\title{
Description of Minimal Entropy Hellinger Sigma Martingale Density of Order One, Order $q$ and Order Zero
}

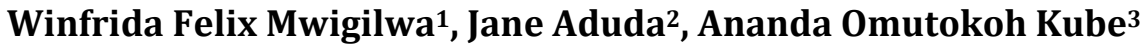 \\ ${ }^{1}$ Department of Mathematics, Pan African University Institute of Basic Sciences, Technology and Innovation, Nairobi, Kenya \\ ${ }^{2}$ Department of Mathematics and Actuarial Sciences, Jomo Kenyatta, University of Agriculture and Technology, Nairobi, Kenya \\ ${ }^{3}$ Department of Mathematics and Actuarial Science, Kenyatta University, Nairobi, Kenya \\ Email: winfridamwigilwa184@gmail.com, jaduda@jkuat.ac.ke, oakube3@googlemail.com
}

How to cite this paper: Mwigilwa, W.F., Aduda, J. and Kube, A.O. (2021) Description of Minimal Entropy Hellinger Sigma Martingale Density of Order One, Order $q$ and Order Zero. Journal of Mathematical Finance, 11, 528-553.

https://doi.org/10.4236/jmf.2021.113030

Received: July 17, 2021

Accepted: August 21, 2021

Published: August 24, 2021

Copyright $\odot 2021$ by author(s) and Scientific Research Publishing Inc. This work is licensed under the Creative Commons Attribution International License (CC BY 4.0).

http://creativecommons.org/licenses/by/4.0/

\begin{abstract}
Generally in this paper, we show how the new version of parameter $U_{2} \in \tilde{\mathcal{P}}$ in Jacod decomposition will change an expression of entropy-Hellinger process of order one, order $q$ and order zero and consequently an equation of minimal entropy Hellinger sigma martingale density for all orders. This is because even the measurable function $\tilde{W} \in \tilde{\mathcal{P}}$ which is an important parameter of an equation of minimal martingale density changes. In order to get a required parameter $\tilde{W} \in \tilde{P}$, we introduce the function $m=f_{t}-1 \in \tilde{\mathcal{P}}$ during our calculation for all orders. The result is different to order zero because we failed to get an equation of minimal entropy-Hellinger sigma martingale density of order zero.
\end{abstract}

\section{Keywords}

Sigma Martingale Density, Jacod Decomposition, Entropy-Hellinger Process, Compensator, Minimal Entropy-Hellinger Sigma Martingale Density

\section{Introduction}

Hellinger processes concepts have been interested in the part of probability theory which addresses the notion of distance between two probability measures [1]. This method is explicitly described in time and arises from the dynamic approach to the Kakutani Hellinger distance between two probability measures. We can say in general Hellinger processes measure an infinitesimal rate of separation of two measures: an equivalent martingale measure $(Q)$ and a physical martingale measure $(P)$. There is an intimate relationship between this entropy-Hellinger 
process and a relative entropy which is used also as a measure of the distance between two probability measures although it is not a metric. In our case, we are going to concentrate on the entropy-Hellinger process which is expressed as a non decreasing process of a jump of local martingale of Jacod decomposition. In this entropy-Hellinger process it is possible to describe the variation of entropyHellinger martingale measure or its entropy-Hellinger martingale density process with respect to the variation of market's factors or parameters [2]. Moreover, the entropy-Hellinger martingale measure has a property of being robust with respect to stopping time. The minimal entropy-Hellinger martingale measure is based on minimizing the entropy-Hellinger process for a nonnegative DoleansDade exponential local martingale [3].

In the research papers [3] and [4] the description of minimal entropy Hellinger local martingale density is done on the Jacod decomposition of the following form $N=\beta \cdot S^{c}+W \star(\mu-v)+g \star \mu+N^{\prime}$ where

$W=U+\frac{\hat{U}}{1-a} \mathbf{1}_{\{a<1\}} \in \mathcal{G}_{\text {loc }}^{1}(\mu)$. This description of minimal entropy Hellinger local martingale density was used by [5] and [6] as sigma martingale density to express its entropy-Hellinger process and also a description of a minimal entropy Hellinger sigma martingale density under its required set when there is a change of probability measure done by [5] and [6]. According to [7] and [8] we can choose a version of $U$ such that if $\Delta N>-1$ then also $U>-1$ and setting $U=f-1$, this gives $W=f-1+\frac{\hat{f}-a}{1-a} \mathbf{1}_{\{a<1\}}$.

This study gives other expression of entropy-Hellinger process for positive sigma martingale of order one, order $q$ and order zero when

$N=\beta \cdot S^{c}+W \star(\mu-v)+g \star \mu+N^{\prime}$ and $W=f-1+\frac{\hat{f}-a}{1-a} \mathbf{1}_{\{a<1\}}$. Further, we find an equation of minimal entropy Hellinger sigma martingale density of order one, order $q$ and order zero.

\section{Methods of Equivalent Martingale Measure}

There has been an increasing interest in providing quantitative approaches to the portfolio optimization, pricing and hedging of contingent claims since the emergence of modern finance. Since martingale methods have been introduced by [9] and [10], these problems can be addressed very elegantly in complete models. The major key tool they came up with in providing solutions of these problems to the complete model is the unique equivalent martingale measure. According to [11], by computing conditional expectations of some random variables under this unique equivalent martingale measure it yields important part of the solution: the unique arbitrage free contingent claim value in the derivative pricing problem, the value of a replicating portfolio in the hedging problem and optimal wealth process in utility maximization problems.

However, in the situation of incomplete markets the case is more involved because one is facing a mathematical and a conceptual problem. Mathematically, 
because the use of martingale methods is complicated by the fact that, there are infinitely many equivalent martingale measures. The question that an investor faces in this case, is concerned with an appropriate equivalent martingale measure to be chosen. Thus many research works have proposed several methods to be used to choose the appropriate equivalent martingale measure.

The first class of methods which were proposed deal with some sort of distance minimization between an equivalent martingale measure and physical probability measure. An appropriate martingale measure is the one which has a smallest distance compared to the other martingale measures. The selection of appropriate martingale measure with the smallest distance compared to the other is due to different optimization criteria for the set of all equivalent martingale measures. For instance, minimal martingale measure proposed by [12] [13] [14] and [15]. Mean-Variance Martingale measure proposed by [16] and [17]. The minimal entropy martingale measure defined and introduced by [18] [19] [20] and [21]. Minimax martingale measure introduced by [22] and [23]. According to [24], all the above martingale measures were defined and characterised in a way that is far from practical use except in the case of market driven by Levy processes. Because of that reason [25] derived the minimal Hellinger martingale measure for a market driven by processes with independent increments.

The second class of methods consists of concepts of utility based pricing. We can call this method an equilibrium-based pricing approach. This method is investigated by [26]. According to [26] if option pricing is inculcated in a utility maximization framework, then a unique martingale measure emerges in a very natural way. Fair price of the contingent claim was based on the assumptions that an investor is willing to divert only an infinitely large amount of his initial amount of wealth to sell (or purchase) a claim. The concept of utility based pricing is related to utility maximization and its dual problem was discussed by [22] [23] [27] and [28] in different ways and in different frameworks. For example, in a continuous time diffusion incomplete model [27] showed that one way to handle the utility maximization problem from terminal wealth is to consider the dual problem. This dual problem can be formulated as a minimization problem over the set of martingale measures. In order to find the solution of this minimization problem, martingale methods were used to recover the solution of the original problem. The minimum is attained by an element in the martingale measure set. The derivation of martingale measures of these research works were based in a static manner which means that the evolution over time of these extremal solutions is not described in these works.

Because of that reason a dynamic version of these works were proposed by [11] [29], and [30]. For example the problem of hedging which is based on utility maximization solved by [29]. The utility maximization solved by [29] was different from expected utility of consumption or terminal wealth. This is because it was done as optimization of the expected utility of gains over infinitesimal time intervals. One advantage of it is that there is no dependence on a terminal date. In [30] a problem based on the utility method for the valuation and hedg- 
ing of non replicable contingent claims was solved. The issuer's valuation process and hedging strategy are defined via utility indifference arguments. The density process used in this paper is written in a particular form in order to get a required solution. In this dynamic version a derivation of a portfolio or a critical martingale measure is independent of the initial/or maturity time investment.

On this dynamic concept another method which was proposed is known as entropy-Hellinger process. This method is expressed as a jump of local martingale of Jacod decomposition [24]. The first paper to create the concept of entropy-Hellinger process which is expressed as local martingale of Jacod decomposition is [24]. The author obtained the minimal entropy-Hellinger local martingale density for the case where the discounted price process is bounded and it has a property of quasi-left continuous. Because the discounted price process is bounded there was no need to introduce a truncation function on its decomposition function. The results of [24] were extended by [3] by finding the minimal entropy-Hellinger local martingale density when the discounted price process is unbounded and may have predictable jumps. In this paper there is an introduction of the truncation function due to unbounded condition of discounted price. The results of [3] was extended by [4] where the author created the concept of Hellinger process of order $q$ for local martingale densities and give its properties. The researcher obtained the minimal-entropy Hellinger local martingale densities of order $q$.

The concept of minimal entropy Hellinger sigma martingale density is introduced by [5] [6]. According to [6] the existence of minimal entropy Hellinger sigma martingale density is due to the fact that, when there is minimal sigma martingale density $\tilde{Z}$ which can minimize the entropy-Hellinger process over its defined set. One of the important difference between the minimal entropy Hellinger sigma martingale density and minimal entropy Hellinger local martingale density is the kind of set in which the minimization is taking place and necessary and sufficient conditions for an exponential local martingale to be a local martingale density or a sigma martingale density.

This paper contributes to the existing literature because we show how the entropy-Hellinger process for positive sigma martingale of order one, order $q$ and order zero can be modified when we have another version of its important parameter $U$. Also we proved that even a description of minimal entropy-Hellinger sigma martingale density of all orders is going to change when we are solving minimization problems based on those entropy-Hellinger processes.

The rest of the paper is organized as follows. Section 2, discusses an expression of entropy Hellinger process for a positive sigma martingale of order one followed by a solution of minimization problem based on entropy Hellinger process of sigma martingale density of order one in Section 3. In Section 4, we provide an expression of entropy Hellinger process for a positive sigma martingale of order $q$ followed by a solution of minimization problem based on entropy-Hellinger process of sigma martingale density of order $q$ in Section 5. A discussion on an expression of entropy-Hellinger process for a positive sigma martingale of order zero is in Section 6. In Section 7, we provide a solution of the 
minimization problem based on the entropy-Hellinger process of sigma martingale density of order zero.

\section{An Expression of Entropy Hellinger Process for a Positive Sigma Martingale of Order One}

According to [6] a $\sigma$-martingale density for a discounted price $S$ is any positive local martingale $Z$ such that $Z S$ is a $\sigma$-martingale. If furthermore $Z S$ is locally integrable then $Z$ is called a local martingale density. This means according to Ito's formula we deduce that $Z S$ is a $\sigma$-martingale if and only $S+[S, N]$ is a $\sigma$-martingale.

According to [5] if $Z=\mathcal{E}(N)$ is a positive local martingale and $\left(\beta, U, g, N^{\prime}\right)$ be the Jacod component of $N$. Then $Z$ is a $\sigma$-martingale density for $(S, P)$ if and only if

$$
\begin{gathered}
\int|x(1+U(x)-h(x))| F(\mathrm{~d} x)<\infty \\
b \cdot A+c \beta \cdot A+(x-h(x)+x U(x)) \star v=0
\end{gathered}
$$

Furthermore, if $Z$ is a $\sigma$-martingale density for $(S, P)$ then the following holds

$$
\int x(1+U(x)) F(\mathrm{~d} x) \Delta A=0
$$

According to [3] [4] [24] [31] if we let $N \in \mathcal{M}_{0, \text { loc }}(P)$ such that $1+\Delta N \geq 0$. Then there exist a predictable and $S^{c}$-integrable process $\beta$, some $N^{\prime} \in \mathcal{M}_{0, l o c}(P)$ with $\left[N^{\prime}, S\right]=0$ and functionals $U \in \tilde{\mathcal{P}}$ and $g \in \tilde{\mathcal{O}}$ such that $\int_{0}^{T} \int_{\mathbb{R}^{d}}|U| v(\mathrm{~d} x, \mathrm{~d} t)<+\infty$ and $\left(\sum_{0<s \leq t} g\left(s, \Delta S_{s}\right)^{2} \mathbf{1}_{\Delta S_{s} \neq 0}\right)^{\frac{1}{2}}$ and $\mathcal{M}_{\mu}^{P}(g \mid \mathcal{P})=0$.

$$
N=\beta \cdot S^{c}+W \star(\mu-v)+g \star \mu+N^{\prime} \quad \text { where } W=U+\frac{\hat{U}}{1-a}
$$

The above Equation (4) is called the Jacod decomposition with parameters $\left(\beta, U, g, N^{\prime}\right)$.

According to [31] if $W \in \mathcal{G}_{\text {loc }}^{1}(\mu)$, Then the stochastic integral $W \star(\mu-v)$ of $W$ with respect to $(\mu-v)$ is the unique element of $\mathcal{M}_{\text {loc }}^{d}$ satisfying

$$
\Delta[W \star(\mu-v)]=\bar{W}_{t}(w) \text { where } \bar{W}_{t}(w)=W(w, t) \mathbf{1}_{\{\Delta S \neq 0\}}-\hat{W}_{t}(w, t)
$$

Also according to [31] If $g \in \mathcal{H}_{\text {loc }}^{1}(\mu)$ where

$\mathcal{H}_{\text {loc }}^{1}(\mu)=\left\{\mathcal{M}_{\mu}^{P}(g \mid \mathcal{P})=0:\right.$ and $\left.\left(g^{2} \star \mu\right)^{\frac{1}{2}} \in \mathcal{A}_{\text {loc }}\right\}$, Then the stochastic integral

$g \star \mu$, of $g$ with respect to $\mu$ is the unique element of $\mathcal{M}_{\text {loc }}^{d}$ satisfying

$$
\Delta(g \star \mu)_{t}=g(t, w) \mathbf{1}_{\{\Delta S=0\}}
$$

Therefore from above Equations (4), (5) and (6) we can build the equation of jump of Jacod decomposition $N$ as follows

$$
\Delta N_{t}=\left(U_{t}\left(\Delta S_{t}\right)+g_{t}\left(\Delta S_{t}\right)\right) \mathbf{1}_{\Delta S_{t} \neq 0}+\left(\Delta N_{t}^{\prime}-\frac{\hat{U}_{t}}{1-a_{t}}\right) \mathbf{1}_{\Delta S_{t}=0}
$$


According to [3] [5] [6] [24], if we let $N \in \mathcal{M}_{0, \text { loc }}$ (P) such that $1+\Delta N \geq 0$, such that the non-decreasing adapted process

$$
V_{t}^{E}(N)=\frac{1}{2}\left\langle N^{c}\right\rangle_{t}+\sum_{0<s \leq t}\left[\left(1+\Delta N_{s}\right) \log \left(1+\Delta N_{s}\right)-\Delta N_{s}\right]
$$

is locally integrable (i.e. $V^{E}(N) \in \mathcal{A}_{\text {loc }}(P)$ ), then its compensator (with respect to the probability $P$ ) is called the entropy-Hellinger process of $N$, denoted by $h_{t}^{E}(N, P)$.

The entropy-Hellinger process of $N$ with respect to probability measure $P$ denoted by $\left(h_{t}^{E}(N, P)\right)$ is the same as of sigma density $Z \in \mathcal{Z}_{\text {loc }}^{e}$ denoted by $\left(h_{t}^{E}(Z, P)\right)$ and of equivalent probability measure $Q \in \mathbb{P}_{a}$ denoted by $\left(h_{t}^{E}(Q, P)\right)$.

According to [7] [8] from Equation (5) where $\beta$ is $S^{c}$ integrable, and $W=U+\frac{\hat{U}}{1-a} \mathbf{1}_{\{a<1\}} \in \mathcal{G}_{\text {loc }}^{1}(\mu), g \in \mathcal{H}_{\text {loc }}^{1}(\mu)$ and $N^{\prime}$ is a local $P$-martingale null at 0 with $\left[S, N^{\prime}\right]=0$

If $\Delta N>-1$ then also $U>-1$, if we have a new version of $U=f-1$ then this gives $W=f-1+\frac{\hat{f}-1}{1-a} \mathbf{1}_{\{a<1\}}$.

Therefore we are going to have the following equation of jump of Jacod decomposition:

$$
\Delta N=(f+g-1) \mathbf{1}_{\{\Delta S \neq 0\}}+\left(\Delta N^{\prime}-\frac{\hat{f}-a}{1-a}\right) \mathbf{1}_{\{\Delta S=0\}}
$$

Proposition 1 (The entropy-Hellinger process of order 1)

The Hellinger process of order 1 for $N$ when $W=f-1+\frac{\hat{f}-1}{1-a} \mathbf{1}_{\{a<1\}}$ is equal to

$$
\begin{aligned}
h^{E}(Z, P)= & \frac{1}{2} \beta^{\mathrm{T}} c \beta \cdot A+[f \ln f-(f-1)] \star v \\
& +\sum_{0<s \leq t}\left[\frac{1-\hat{f}}{1-a} \ln \left(1-\frac{\hat{f}-a}{1-a}\right)+(\hat{f}-a)\right] \\
& +f \mathcal{M}_{\mu}^{P}\left(\left(1-\frac{g}{f}\right) \ln \left(1+\frac{g}{f}\right)-\frac{g}{f} \mid \tilde{P}\right) \star v+K
\end{aligned}
$$

Proof

To prove the Equation (10) we are going to apply the same method used in [3].

From (4) and (9), lets define $\bar{g}=\frac{g}{f} \mathbf{1}_{\{f>0\}}$

Then we are going to have the following assertions

$$
\begin{aligned}
& g \mathbf{1}_{\{f=0\}}=0 \\
& \Delta N^{\prime} \mathbf{1}_{\{\hat{f}-a=1-a\}}=0
\end{aligned}
$$

From Equation (8)

$$
1+\Delta N=(f+g) \mathbf{1}_{\{\Delta S \neq 0\}}+\left(1+\Delta N^{\prime}-\frac{\hat{f}-a}{1-a}\right) \mathbf{1}_{\{\Delta S=0\}}
$$


Then

$$
\begin{aligned}
& f+g>0 \\
& 1+\Delta N^{\prime}-\frac{\hat{f}-a}{1-a}>0
\end{aligned}
$$

By taking the conditional expectation under $\mathcal{M}_{\mu}^{P}$ with respect to $\tilde{\mathcal{P}}$ and a predictable projection of above equations we are going to get

$$
\begin{aligned}
& f>0 \\
& 1-\frac{\hat{f}-a}{1-a}>0
\end{aligned}
$$

From Equation (12)

$$
\begin{aligned}
& \sum_{0<s \leq t}\left[\left(1+\Delta N_{s}\right) \ln \left(1+\Delta N_{s}\right)-\Delta N_{s}\right] \\
= & {[(f+g) \ln (f+g)-(f+g-1)] \star \mu } \\
& +\sum\left[\left(1-\frac{\hat{f}-a}{1-a}+\Delta N\right) \ln \left(1-\frac{\hat{f}-a}{1-a}+\Delta N\right)+\frac{\hat{f}-a}{1-a}-\Delta N^{\prime}\right] \mathbf{1}_{\{\Delta S=0\}}
\end{aligned}
$$

Thanks to the assertions above on the Equation (11).

$$
\begin{aligned}
V(N, P)= & \frac{1}{2} \beta^{\mathrm{T}} c \beta \cdot A+[f \ln f-(f-1)] \star \mu \\
& +\sum_{0<s \leq t}\left[\left(1-\frac{\hat{f}-a}{1-a}\right) \ln \left(1-\frac{\hat{f}-a}{1-a}\right)+\frac{\hat{f}-a}{1-a}\right] \mathbf{1}_{\Delta S=0} \\
& +f\left[\left(1+\frac{g}{f}\right) \ln \left(1+\frac{g}{f}\right)-\frac{g}{f}\right] \star \mu+\frac{1}{2}\left\langle N_{t}^{\prime c}\right\rangle \\
& \left.\left.+\sum_{0<s \leq t}\left[\left(1-\frac{\hat{f}-a}{1-a}\right)\left(\left[1+\frac{\Delta N^{\prime}}{\hat{f}-a}\right) \ln \left(1+\frac{\Delta N^{\prime}}{1-a}\right)-\frac{\hat{f}-a}{1-\frac{\hat{f}}{1-a}}\right)\right] \frac{\Delta N^{\prime}}{1-a}\right)\right] \\
& +(g \ln f) \star \mu+\sum_{0<s \leq t}\left(1-\frac{\hat{f}-a}{1-a}\right) \Delta N^{\prime} \mathbf{1}_{\{\Delta S=0\}}
\end{aligned}
$$

The dual predictable projection of the two last terms in the RHS of the Equation (14) of the above equation vanish. We are going to remain with

$$
\begin{aligned}
& V(N, P)= \frac{1}{2} \beta^{\mathrm{T}} c \beta \cdot A+[f \ln f-(f-1)] \star \mu \\
&+\sum_{0<s \leq t}\left[\left(1-\frac{\hat{f}-a}{1-a}\right) \ln \left(1-\frac{\hat{f}-a}{1-a}\right)+\frac{\hat{f}-a}{1-a}\right] \mathbf{1}_{\Delta S=0} \\
&+f\left[\left(1+\frac{g}{f}\right) \ln \left(1+\frac{g}{f}\right)-\frac{g}{f}\right] \star \mu+\frac{1}{2}\left\langle N_{t}^{\prime c}\right\rangle \\
&+\sum_{0<s \leq t}\left[\left(1-\frac{\hat{f}-a}{1-a}\right)\left(\left(1+\frac{\Delta N^{\prime}}{\hat{f}-a}\right) \ln \left(1+\frac{\Delta N^{\prime}}{1-a}\right)-\frac{\Delta N^{\prime}}{1-a}\right)\right] \\
&\left.\left.1-\frac{\hat{f}-a}{1-a}\right)\right]
\end{aligned}
$$


Taking dual predictable projection we are going to have the following equation

$$
\begin{aligned}
h^{E}(Z, P)= & \frac{1}{2} \beta^{\mathrm{T}} c \beta \cdot A+[f \ln f-(f-1)] \star v \\
& +\sum_{0<s \leq t}\left[\left(1-\frac{\hat{f}-a}{1-a}\right) \ln \left(1-\frac{\hat{f}-a}{1-a}\right)+\frac{\hat{f}-a}{1-a}\right](1-a) \\
& +f \mathcal{M}_{\mu}^{P}\left(\left(1+\frac{g}{f}\right) \ln \left(1+\frac{g}{f}\right)-\frac{g}{f} \mid \tilde{P}\right) \star v+K
\end{aligned}
$$

where $K$ is a predictable projection of the

$$
\frac{1}{2}\left\langle N_{t}^{\prime c}\right\rangle+\sum_{0<s \leq t}\left[\left(1-\frac{\hat{f}-a}{1-a}\right)\left(\left(1+\frac{\Delta N^{\prime}}{1-\frac{\hat{f}-a}{1-a}}\right) \ln \left(1+\frac{\Delta N^{\prime}}{1-\frac{\hat{f}-a}{1-a}}\right)-\frac{\Delta N^{\prime}}{1-\frac{\hat{f}-a}{1-a}}\right)\right] .
$$

Then the required entropy-Hellinger process is

$$
\begin{aligned}
h^{E}(Z, P)= & \frac{1}{2} \beta^{\mathrm{T}} c \beta \cdot A+[f \ln f-(f-1)] \star v \\
& +\sum_{0<s \leq t}\left[\frac{1-\hat{f}}{1-a} \ln \left(1-\frac{\hat{f}-a}{1-a}\right)+\hat{f}-a\right] \\
& +f \mathcal{M}_{\mu}^{P}\left(\left(1+\frac{g}{f}\right) \ln \left(1+\frac{g}{f}\right)-\frac{g}{f} \mid \tilde{P}\right) \star v+K
\end{aligned}
$$

which is the same as (10).

From Equation (4), If we set $N^{1}=\beta \cdot S^{c}+W \star(\mu-v)$ and $Z^{1}=\mathcal{E}\left(N^{1}\right)$. If $Z S$ is a $\sigma$-martingale. then $Z^{1} S$ is also a $\sigma$-martingale.

Since we are going to focus on $\sigma$-martingales with finite entropy. The set of these measures is given by the following sets. ([5] and [6]).

$$
\begin{gathered}
\mathcal{M}_{f}^{e}(S)=\left\{Q \in \mathbb{P}_{e} \mid S \in \mathcal{M}_{\sigma}(Q) \text { and } E\left[\frac{\mathrm{d} Q}{\mathrm{~d} P} \log \left(\frac{\mathrm{d} Q}{\mathrm{~d} P}\right)\right]<+\infty\right\} \\
\mathcal{Z}_{\text {loc }}^{e}(S)=\left\{Z \in \mathcal{M}_{\text {loc }}(P) \mid Z>0, Z \log (Z) \text { is locally integrable, } Z S \in \mathcal{M}_{\sigma}(P)\right\}
\end{gathered}
$$

The minimization problem of $\min _{Z \in \mathcal{Z}_{e}, \text { loc }} h^{E}(Z, P)$ is equivalent to minimize the Hellinger process over the set of densities that have the following predictable representation ( $g=0$ and $N^{\prime}=0$ ).

Therefore we are going to have the following minimization entropy-Hellinger process.

$$
\begin{aligned}
h^{E}(Z, P)= & \frac{1}{2} \beta^{\mathrm{T}} c \beta \cdot A+[f \ln f-(f-1)] \star v \\
& +\sum_{0<s \leq t}\left[\frac{1-\hat{f}}{1-a} \ln \left(1-\frac{\hat{f}-a}{1-a}\right)+\hat{f}-a\right]
\end{aligned}
$$

\section{Solution of Minimization Problem Based on Entropy Hellinger Process of Sigma Martingale Density of Order \\ One}

Theorem 2 According to [3], in order to have minimization solution of (20) 
we have an assumption of discounted price process

$$
\int_{|x|>1}|x| \exp \left(\lambda^{\mathrm{T}} x\right) F(\mathrm{~d} x)<+\infty
$$

and the solution to

$$
\min _{Z \in \mathcal{Z}_{a, l o c}} h^{E}(Z, P)
$$

exists and is given by

$$
\begin{aligned}
& \tilde{Z}=\mathcal{E}(N)>0 \\
& \tilde{N}_{t}=\tilde{\beta} \cdot S_{t}^{c}+\tilde{W} \star(\mu-v)
\end{aligned}
$$

where

$$
\begin{aligned}
& \tilde{W}_{t}(x)=\frac{\exp \left(\tilde{\lambda}_{t}^{\mathrm{T}} x\right)-1}{1-a_{t}+\int \exp \left(\tilde{\lambda}_{t}^{\mathrm{T}} x\right) v(t \times(\mathrm{d} x))} \\
& \tilde{\beta}_{t}=\tilde{\lambda}_{t} \mathbf{1}_{\left\{\Delta A_{t}=0\right\}}
\end{aligned}
$$

where $\tilde{\lambda}_{t} \mathbf{1}_{\left\{\Delta A_{t} \neq 0\right\}}$ is a root for

$$
\int \exp (x \lambda) \times v(w ; t \times(\mathrm{d} x))=0
$$

while $\tilde{\lambda}_{t} \mathbf{1}_{\left\{\Delta A_{t}=0\right\}}$ is a root for

$$
0=G^{E}(w, t, \lambda)=b_{t}(w)+c_{t}(w) \lambda+\int\left(\mathrm{e}^{x \lambda} x-h(x)\right) F_{t}(w, \mathrm{~d} x)
$$

Since we know that $Z=\mathcal{E}(N)=\mathcal{E}\left(\beta \cdot S^{c}+W \star(\mu-v)\right)$. We are going to minimize the following equation

$$
\min _{(f, \beta)}\left(\frac{1}{2} \beta^{\mathrm{T}} c \beta \cdot A+[f \ln f-(f-1)] \star v+\sum_{0<s \leq t}\left[\frac{1-\hat{f}}{1-a} \ln \left(1-\frac{\hat{f}-a}{1-a}\right)+\hat{f}-a\right]\right)
$$

By distinguishing the cases where $\Delta A=0$ and the case where $\Delta A \neq 0$, this problem can be split into the following two minimization problems. The first problem $(\triangle A=0)$ is defined by

$$
\int \frac{1}{2} \beta^{\mathrm{T}} c \beta \mathrm{d} A+\int[f \ln f-(f-1)] F_{t}(\mathrm{~d} x) \mathrm{d} A
$$

where the minimization is over all couples $(\beta, f)$ satisfying

$$
b+c \beta+\int(x-h(x)+x f) F_{t}(\mathrm{~d} x)=0
$$

The second problem $(\Delta A \neq 0$ ) is defined as follows

$$
\int[f \ln f-(f-1)] v_{t}(\mathrm{~d} x)+\sum_{0<s \leq t}\left[\frac{1-\hat{f}}{1-a} \ln \left(1-\frac{\hat{f}-a}{1-a}\right)+\hat{f}-a\right]
$$

where the minimization is over the functional $f$ such that

$$
\int x f v_{t}(\mathrm{~d} x)=0
$$

The conditions (24) and (26) correspond to the conditions given in above theorem and conditions which is known for given for a local martingale to be a sigma martingale density Equations (2) and (3) 


\subsection{Solving Euler-Lagrange Equation of the First Problem}

Euler-Lagrange equation of the first problem $(\Delta A=0)$ which is the combination of Equations (23) and (24).

$$
\begin{gathered}
L(\beta, f)=\int \frac{1}{2} \beta^{\mathrm{T}} c \beta \mathrm{d} A+\int[f \ln f-(f-1)] F_{t}(\mathrm{~d} x) \mathrm{d} A \\
-\lambda\left(\int b \mathrm{~d} A+\int c \beta \mathrm{d} A+\int(x-h(x)+x f) F_{t}(\mathrm{~d} x) \mathrm{d} A\right) \\
=0 \\
L(\beta, f)=\frac{1}{2} \beta^{\mathrm{T}} c \beta+\int[f \ln f-(f-1)] F_{t}(\mathrm{~d} x) \\
-\lambda\left(b+c \beta+\int(x-h(x)+x f) F_{t}(\mathrm{~d} x)\right) \\
=0 \quad \mathrm{~d} \beta: \beta=\lambda \\
\mathrm{d} f: f=\mathrm{e}^{\lambda x}
\end{gathered}
$$

Since our $W=f-1+\frac{\hat{f}-a}{1-a}$, we must introduce a new function $m=f_{t}-1 \in \tilde{\mathcal{P}}$.

Therefore we are going to have the following function for the first Lagrange equation $m=f_{t}-1=\mathrm{e}^{\lambda x}-1$.

Therefore the description of $\tilde{\beta}$ and $\tilde{f}$ is completely established

$$
\begin{aligned}
& \tilde{\beta}=\tilde{\lambda} \mathbf{1}_{\left\{\Delta A_{t}=0\right\}} \\
& m \mathbf{1}_{\left\{\Delta A_{t}=0\right\}}=f_{t} \mathbf{1}_{\left\{\Delta A_{t}=0\right\}}-1=\exp \left(\lambda^{\mathrm{T}} x \mathbf{1}_{\Delta A_{t}=0}\right)-1
\end{aligned}
$$

where $\lambda$ is the root for $b+c \lambda+\int\left(\mathrm{e}^{\lambda^{\mathrm{T}} x} x-h(x)\right) F_{t}(\mathrm{~d} x)=0$

\subsection{Solving Euler-Lagrange Equation of the Second Problem}

Euler-Lagrange equation of the second problem $(\Delta A \neq 0)$ which is the combination of Equations (25) and (26).

$$
\begin{aligned}
L(f, \phi, \lambda, \alpha)= & \int[f \ln f-(f-1)] v_{t}(\mathrm{~d} x)+\sum_{0<s \leq t}\left[(1-a-\phi) \ln \left(1-\frac{\phi}{1-a}\right)+\phi\right] \\
& -\lambda\left(\int x f v_{t}(\mathrm{~d} x)\right)-\alpha\left(\int f v_{t}(\mathrm{~d} x)-a-\phi\right)
\end{aligned} \quad \begin{aligned}
\mathrm{d} f: \ln f=\lambda x+\alpha \\
\\
\quad f=\mathrm{e}^{\lambda x+\alpha} \\
\mathrm{d} \phi: \ln \left(1-\frac{\phi}{1-a}\right)=\alpha \\
\mathrm{d} \lambda: \int x f v_{t}(\mathrm{~d} x)=0 \\
\mathrm{~d} \alpha: \int x f v_{t}(\mathrm{~d} x)-a-\phi=0
\end{aligned}
$$

Substitute Equations (29) into (31)

$$
\begin{aligned}
& \int \mathrm{e}^{\lambda x+\alpha} x v_{t}(\mathrm{~d} x)=0 \\
& \int \mathrm{e}^{\lambda x} x v_{t}(\mathrm{~d} x)=0
\end{aligned}
$$


Equation (33) is one of the condition of $Z$ to be $\sigma$-martingale density Equation (3).

From Equation (30) we have

$$
\phi=(1-a)\left(1-\mathrm{e}^{\alpha}\right)
$$

Substitute Equations (29) and (34) into Equation (32).

$$
\mathrm{e}^{\alpha}=\frac{1}{1-a+\int \mathrm{e}^{\lambda x} v_{t}(\mathrm{~d} x)}
$$

Substitute Equation (35) into Equation (29)

$$
f_{t}(x)=\frac{\mathrm{e}^{\lambda x}}{1-a+\int \mathrm{e}^{\lambda x} v_{t}(\mathrm{~d} x)}
$$

If you multiply both equation with $v_{t}(\mathrm{~d} x)$ and integrate both equation we are going to have

$$
\int f_{t}(x) v_{t}(\mathrm{~d} x)=\frac{\int \mathrm{e}^{\lambda x} v_{t}(\mathrm{~d} x)}{1-a+\int \mathrm{e}^{\lambda x} v_{t}(\mathrm{~d} x)}
$$

If we add Equations (36) and (37) we are going to have

$$
f_{t}(x)+\hat{f}_{t}(x)=\frac{\mathrm{e}^{\lambda x}+\int \mathrm{e}^{\lambda x} v_{t}(\mathrm{~d} x)}{1-a+\int \mathrm{e}^{\lambda x} v_{t}(\mathrm{~d} x)}
$$

The LHS of Equation (38) which is $f_{t}(x)+\hat{f}_{t}(x)$ makes a function $W=f_{t}(x)+\hat{f}_{t}(x)$ which is not equal to $W=f-1+\frac{\hat{f}-a}{1-a}$. This function $W=f_{t}(x)+\hat{f}_{t}(x)$ makes the $\tilde{W}=\frac{\mathrm{e}^{\lambda x}+\int \mathrm{e}^{\lambda x} v_{t}(\mathrm{~d} x)}{1-a+\int \mathrm{e}^{\lambda x} v_{t}(\mathrm{~d} x)}$.

Since our $W=f-1+\frac{\hat{f}-a}{1-a}$, in order to get the required $W$ and $\tilde{W}$ for minimum entropy-Hellinger sigma martingale density, we must introduce a new equation $m=f_{t}-1 \in \tilde{\mathcal{P}}$.

$$
m=f_{t}-1=\frac{\mathrm{e}^{\lambda x}}{1-a+\int \mathrm{e}^{\lambda x} v_{t}(\mathrm{~d} x)}-1=\frac{\mathrm{e}^{\lambda x} \mathbf{1}_{\Delta A=0}}{1-a+\int \mathrm{e}^{\lambda x} v_{t}(\mathrm{~d} x)}-1
$$

Therefore we have

That is for the second problem.

We can summarize Equations (28) and (29) as follows

$$
m=f_{t}-1=\frac{\mathrm{e}^{\lambda x}}{1-a+\int \mathrm{e}^{\lambda x} v_{t}(\mathrm{~d} x)}-1
$$

From Equation (40) multiply both sides by $v_{t}(\mathrm{~d} x)$ and find integration both sides. Then we are going to get

$$
\frac{\int f_{t}(x) v_{t}(\mathrm{~d} x)-a}{1-a}=\frac{\int \mathrm{e}^{\lambda x} v_{t}(\mathrm{~d} x)-a}{1-a+\int \mathrm{e}^{\lambda x} v_{t}(\mathrm{~d} x)}
$$


By combining Equation (40) and Equation (41) LHS and RHS we get the required minimal entropy Hellinger sigma martingale density

$$
\begin{aligned}
& \tilde{Z}=\mathcal{E}(\tilde{N}), \quad \tilde{N}=\tilde{\beta} \cdot S^{c}+\tilde{W} \star(\mu-v) \\
& \tilde{W}_{t}(x)=\left(\tilde{\gamma}_{t}\right)^{-1}\left(\mathrm{e}^{\tilde{\gamma}_{t}^{\mathrm{T}} x}-1\right), \quad \tilde{\gamma}_{t}=1-a_{t}+\int \mathrm{e}^{\tilde{\gamma}_{t}^{\mathrm{T}} x} v(t, \mathrm{~d} x)
\end{aligned}
$$

which is the same as Equation (21).

A measurable function $W$ in Jacod decomposition depends $U \in \tilde{P}$. We proved that, when we have another version of $U$ it will change a measurable function $W$, an expression of entropy-Hellinger process as well as $\tilde{W}$. In order to get a measurable function $\tilde{W}$ for an equation of minimal entropy-Hellinger sigma martingale density we introduce the function $m=f_{t}-1 \in \tilde{\mathcal{P}}$ and get

$$
\tilde{W}_{t}(x)=\frac{\mathrm{e}^{\tilde{\gamma}_{t}^{\mathrm{T}} x}-1}{1-a_{t}+\int \mathrm{e}^{\tilde{\gamma}_{t}^{\mathrm{T}} x} v(t, \mathrm{~d} x)}
$$

\section{An Expression of Entropy Hellinger Process for a Positive Sigma Martingale of Order $q$}

According to [4] if we let $N \in \mathcal{M}_{0, l o c}(P)$ be such that $(1+\Delta N)>0$ and $q \neq 1$, and such that the nondecreasing adapted process

$$
V_{t}^{q}(N)=\frac{1}{2}\left\langle N^{c}\right\rangle_{t}+\sum_{0<s \leq t} \phi_{q}\left(\Delta N_{s}\right), \quad 0 \leq t \leq T
$$

is locally integrable, then the Hellinger process $H^{(q)}(N, P)$ of order $q \neq 0$ is the dual predictable projection of $V_{t}^{q}(N)$ with respect to $P$.

The function $\phi_{q}(x)$ in Equation (43) is defined as follows

$$
\phi_{q}(x)=\left\{\begin{array}{lc}
\frac{(1+x)^{q}-1-q x}{q(q-1)}, & \text { if } x>-1 \text { and } q \notin 0,1 \\
+\infty & \text { otherwise }
\end{array}\right.
$$

The set of sigma martingale densities which we are interested in is:

$$
\begin{aligned}
\mathcal{Z}_{\text {loc }}^{e}(S)= & \left\{Z=\mathcal{E}(N) \geq 0 \mid N \in \mathcal{M}_{\text {loc }}(P): Z>0 Z \log Z\right. \text { locally integrable, } \\
& \left.Z S \in \mathcal{M}_{\sigma}(P), \phi_{q}(\Delta N) \in L_{\text {loc }}^{1}\right\}
\end{aligned}
$$

As we have seen in the entropy-Hellinger process of order one: The entropy-Hellinger process of $N$ with respect to probability measure $P\left(h^{(q)}(N, P)\right)$ is the same as of sigma density $Z \in \mathcal{Z}_{\text {loc }}^{e}(q)$ denoted by $\left(h_{t}^{(q)}(Z, P)\right)$ and also is the same as of equivalent probability measure $\left(Q \in \mathbb{P}_{a}\right)$ denoted by $\left(h_{t}^{(q)}(Q, P)\right)$.

Proposition 3 (The entropy Hellinger process of order $q$ )

The Hellinger process of order $q$ for $N$ when $W=f-1+\frac{\hat{f}-1}{1-a} \mathbf{1}_{\{a<1\}}$ is equal to

$$
\begin{aligned}
h^{(q)}(N, P)= & \frac{1}{2} \beta^{\mathrm{T}} c \beta \cdot A+\int_{0}^{T} \int_{\mathbb{R}} \phi_{q}(f-1) F_{t}(\mathrm{~d} x)(\mathrm{d} A) \\
& +\sum_{0<s \leq t}(1-a) \phi_{q}\left(-\frac{\hat{f}-a}{1-a}\right)+f^{q} \mathcal{M}_{\mu}^{P}\left(\phi_{q}\left(\frac{g}{f}\right) \mid \tilde{P}\right) \star v+M
\end{aligned}
$$


Proof

We are going to apply the same method used in [4] to prove the above Proposition (3). From Equation (9) we are going to have

$$
\phi_{q}(\Delta N)=\phi_{q}(f+g-1) \mathbf{1}_{\{\Delta S \neq 0\}}+\phi_{q}\left(\Delta N^{\prime}-\frac{\hat{f}-a}{1-a}\right) \mathbf{1}_{\{\Delta S=0\}}
$$

Since $1+\Delta N_{t}>0$, we derive

$$
f+g>0, \quad 1+\Delta N^{\prime}-\frac{\hat{f}-a}{1-a}>0
$$

Taking the conditional expectation under $\mathcal{M}_{\mu}^{P}$ with respect to $\tilde{\mathcal{P}}$ in the first inequality and a predictable projection in the second one we are going to have

$$
\begin{aligned}
& f>0, \quad 1-\frac{\hat{f}-a}{1-a}>0 \\
& g \mathbf{1}_{\{f=0\}}=0, \quad \Delta N^{\prime} \mathbf{1}_{\{1-a=\hat{f}-a\}}=0
\end{aligned}
$$

As from [4] we first compute the compensator of $\Psi=\sum \phi_{q}(f+g-1) \mathbf{1}_{\{\Delta S \neq 0\}}$. Therefore

$$
\phi_{q}(f+g-1)=\phi_{q}(f-1)+f^{q} \phi_{q}\left(\frac{g}{f}\right)+\left(\frac{f^{q-1}-1}{q-1}\right) g
$$

That means we have the following equation

$$
\sum \phi_{q}(f+g-1) \mathbf{1}_{\{\Delta S \neq 0\}}=\sum\left(\phi_{q}(f-1)+f^{q} \phi_{q}\left(\frac{g}{f}\right)+\left(\frac{f^{q-1}-1}{q-1}\right) g\right) \mathbf{1}_{\{\Delta S \neq 0\}}
$$

From the definition of random measure we have

$$
\phi_{q}(f+g-1) \star \mu=\phi_{q}(f-1) \star \mu+f^{q} \phi_{q}\left(\frac{g}{f}\right) \star \mu+\left(\frac{f^{q-1}-1}{q-1}\right) g \star \mu
$$

The compensator of the LHS of Equation (47) is equal to the sum of the compensators of the RHS of Equation (47).

The compensator of $\phi_{q}(f-1) \star \mu$ is equal to the $\phi_{q}(f-1) \star v=\int_{0}^{T} \int_{\mathbb{R}} \phi_{q}(f-1) F_{t}(\mathrm{~d} x) \mathrm{d} A$

The compensator of $f^{q} \phi_{q}\left(\frac{g}{f}\right) \star \mu$ is given by $f^{q} \mathcal{M}_{\mu}^{P}\left(\phi_{q}\left(\frac{g}{f}\right) \mid \tilde{P}\right) \star v$

The compensator of the last term: We are going to introduce the set $C_{n}=\left[(w, t, x): f^{q-1} \neq n\right]$. Since $\mathcal{M}_{\mu}^{P}(g \mid \tilde{P})=0$, Then the compensator of $\left(1_{C_{n}} \frac{f^{q-1}-1}{q-1}\right) g \star \mu$ is equal to 0.

Therefore the compensator of $\Psi$ is

$$
\int_{0}^{T} \int_{\mathbb{R}} \phi_{q}(f-1) F_{t}(\mathrm{~d} x) \mathrm{d} A+f^{q} \mathcal{M}_{\mu}^{P}\left(\phi_{q}\left(\frac{g}{f}\right) \mid \tilde{P}\right) \star v
$$

Now we compute the compensator of 


$$
\digamma=\sum \phi_{q}\left(\Delta N^{\prime}-\frac{\hat{f}-a}{1-a}\right) \mathbf{1}_{\{\Delta S=0\}}
$$

Then

$$
\begin{aligned}
\phi_{q}\left(\Delta N^{\prime}-\frac{\hat{f}-a}{1-a}\right)= & \phi_{q}\left(-\frac{\hat{f}-a}{1-a}\right)+\left(1-\frac{\hat{f}-a}{1-a}\right) \phi_{q}\left(\frac{\Delta N^{\prime}}{1-\frac{\hat{f}-a}{1-a}}\right) \\
& +\frac{1-\left(\frac{\hat{f}-a}{1-a}\right)^{q-1}-1}{q-1} \Delta N^{\prime}
\end{aligned}
$$

Therefore

$$
\begin{aligned}
& \phi_{q}\left(\Delta N^{\prime}-\frac{\hat{f}-a}{1-a}\right) \mathbf{1}_{\{\Delta S=0\}} \\
& =\phi_{q}\left(-\frac{\hat{f}-a}{1-a}\right) \mathbf{1}_{\{\Delta S=0\}}+\left(1-\frac{\hat{f}-a}{1-a}\right) \phi_{q}\left(\frac{\Delta N^{\prime}}{\left.1-\frac{\hat{f}-a}{1-a}\right)}\right) \mathbf{1}_{\{\Delta S=0\}} \\
& +\frac{1-\left(\frac{\hat{f}-a}{1-a}\right)^{q-1}-1}{q-1} \Delta N^{\prime} \mathbf{1}_{\{\Delta S=0\}}
\end{aligned}
$$

The compensator of the LHS of Equation (49) is equal to the sum of the compensators of the RHS of Equation (49).

The compensator of $\sum_{0<s \leq t} \phi_{q}\left(-\frac{\hat{f}-a}{1-a}\right)$ is equal to

$$
\sum_{0<s \leq t}(1-a) \phi_{q}\left(-\frac{\hat{f}-a}{1-a}\right)
$$

Let the compensator of $\left(1-\frac{\hat{f}-a}{1-a}\right) \phi_{q}\left(\frac{\Delta N^{\prime}}{1-\frac{\hat{f}-a}{1-a}}\right)$ be equal to $M$

The compensator of the process $\frac{1-\left(\frac{\hat{f}-a}{1-a}\right)^{q-1}-1}{q-1} \Delta N^{\prime}$ is equal to 0.

Therefore the compensator of $\digamma$ is

$$
\sum_{0<s \leq t}(1-a) \phi_{q}\left(-\frac{\hat{f}-a}{1-a}\right)+M
$$

Therefore by combining Equation (48) and Equation (50) we get Equation (45).

From Jacod decomposition Equation (4) if we set $N^{1}=\beta \cdot S^{c}+W \star(\mu-v)$ 
and $Z^{1}=\mathcal{E}\left(N^{1}\right)$. If $Z S$ is a $\sigma$-martingale. then $Z^{1} S$ is also a $\sigma$-martingale because $N^{\prime}$ preserves the martingales property for $S$,

Then $\mathcal{Z}_{e, \sigma}^{r, 1}(q) \subseteq \mathcal{Z}_{e, \sigma}(q)$

where $\mathcal{Z}_{e, \sigma}^{r, 1}(q)=\left\{Z=\mathcal{E}\left(N^{\prime}\right) \mid \mathcal{E}(N) \in \mathcal{Z}_{e, \sigma}(q)\right\}$.

Therefore for the minimization problem

$$
\min _{Z \in \mathcal{Z}_{e, l o c}} h^{(q)}(Z, P)=\min _{Z \in \mathcal{Z}_{e, l o c}^{, 1}} h^{(q)}(Z, P)
$$

we are going to have the following equation for the hellinger process.

$$
\begin{aligned}
h^{(q)}(N, P)= & \frac{1}{2} \beta^{\mathrm{T}} c \beta \cdot A+\int_{0}^{T} \int_{\mathbb{R}} \phi_{q}(f-1) F_{t}(\mathrm{~d} x)(\mathrm{d} A) \\
& +\sum_{0<s \leq t}(1-a) \phi_{q}\left(-\frac{\hat{f}-a}{1-a}\right)
\end{aligned}
$$

\section{Solution of Minimization Problem Based on Entropy Hellinger Process of Sigma Martingale Density of Order $q$}

Theorem 4 According to [4] for a solution of Equation (51) to exist, we have an assumption of discounted price proces

$$
\int\left|\left(1+\lambda^{\mathrm{T}} x\right)^{\frac{1}{q-1}} x-h(x)\right| F(\mathrm{~d} x)<+\infty
$$

and a solution of Equation (51) exists and is given by

$$
\begin{aligned}
& Z^{(q)}=\mathcal{E}\left(N^{(q)}\right) \\
& N^{(q)}=\beta^{(q)} \cdot S^{c}+W^{(q)} \star(\mu-v)
\end{aligned}
$$

where

$$
\begin{aligned}
& \beta_{t}^{(q)}=\frac{1}{q-1} \tilde{\lambda}_{t} \\
& W^{(q)}(t, x)=\frac{\left(1+\tilde{\lambda}_{t}^{\mathrm{T}} x\right)^{\frac{1}{q-1}}-1}{1-a_{t}+\int\left(1+\tilde{\lambda}_{t}^{\mathrm{T}} x\right)^{\frac{1}{q-1}} v_{t}(\mathrm{~d} x)}
\end{aligned}
$$

Since we know that $Z^{(q)}=\mathcal{E}\left(N^{(q)}\right)=\mathcal{E}\left(\beta^{(q)} \cdot S^{c}+W^{(q)} \star(\mu-v)\right)$, we are going to minimize the following equation

$$
\min _{\left\{f_{t}(x), \beta\right\}}\left(\frac{1}{2} \beta^{\mathrm{T}} c \beta \cdot A+\int_{0}^{T} \int_{\mathbb{R}} \phi_{q}(f-1) F_{t}(\mathrm{~d} x)(\mathrm{d} A)+\sum_{0<s \leq t}(1-a) \phi_{q}\left(-\frac{\hat{f}-a}{1-a}\right)\right)
$$

By distinguishing the cases where $\Delta A=0$ and the case where $\Delta A \neq 0$, this problem can be split into the following two minimization problems.

The first problem $\Delta A=0$ is defined by

$$
\frac{1}{2} \beta^{\mathrm{T}} c \beta+\int_{0}^{T} \int_{\mathbb{R}} \phi_{q}(f-1) F_{t}(d x)
$$

where the minimization is over all couples $\left(\beta, f_{t}(x)\right)$ satisfying 


$$
b+c \beta+\int(x-h(x)+x(f-1)) F_{t}(\mathrm{~d} x)
$$

The second problem $\Delta A \neq 0$ is defined as follows

$$
\int_{0}^{T} \int_{\mathbb{R}} \phi_{q}(f-1) F_{t}(\mathrm{~d} x)(\mathrm{d} A)+\sum_{0<s \leq t}(1-a) \phi_{q}\left(-\frac{\hat{f}-a}{1-a}\right)
$$

where the minimization is over the function $f_{t}(x)$ such that

$$
\int f_{t}(x) x v_{t}(\mathrm{~d} x)=0
$$

\subsection{Solving Euler-Lagrange Equation of the First Problem}

Euler-Lagrange equation of the first problem on $\Delta A=0$ which is a combination of (56) and Equation (57)

$$
\begin{aligned}
& L(\beta, f(x), \lambda)=\frac{1}{2} \beta^{\mathrm{T}} c \beta+\int_{0}^{T} \int_{\mathbb{R}} \frac{f^{q}-1-q(f-1)}{q(q-1)} \\
& -\lambda\left(b+c \beta+\int(x-h(x)+x(f-1)) F_{t}(\mathrm{~d} x)\right) \\
& \mathrm{d} \beta: \quad \beta c=\lambda c \\
& \mathrm{~d} f: \quad f=(\lambda x+1)^{\frac{1}{q-1}} \beta=\lambda \\
& \mathrm{d} \lambda:\left(b+c \beta+\int(x-h(x)+x(f-1)) F_{t}(\mathrm{~d} x)\right)=0
\end{aligned}
$$

Therefore we are going to have the following

$$
\begin{aligned}
& \tilde{\beta}=\lambda \\
& \tilde{f}_{t}(x)=(\lambda x+1)^{\frac{1}{q-1}}
\end{aligned}
$$

When we substitute Equation (60) into Equation (61).

$$
b+c \lambda+\int\left((\lambda x+1)^{\frac{1}{q-1}}-h(x)\right) F_{t}(\mathrm{~d} x)=0
$$

\subsection{Solving Euler-Lagrange Equation of the Second Problem}

The Second equation on $\Delta A \neq 0$ is as follows

$$
\begin{aligned}
L(f(x), \lambda, \rho)= & \int_{0}^{T} \int_{\mathbb{R}} \frac{f^{q}-1-q(f-1)}{q(q-1)} F_{t}(\mathrm{~d} x) \\
& +(1-a) \frac{\left(\frac{1-a-\kappa}{1-a}\right)^{q}-1-q\left(\frac{-\kappa}{1-a}\right)}{q(q-1)} \\
& -\lambda\left(\int x f_{t}(x) F_{t}(\mathrm{~d} x)\right)-\rho\left(\int f_{t}(x) v_{t}(\mathrm{~d} x)-a-\kappa\right)
\end{aligned}
$$

We are going to have the following equations

$$
\begin{array}{ll}
\mathrm{d} f: & f^{q-1}-1=(q-1)(\lambda x+\rho) \\
\mathrm{d} \kappa: & (1-a-\kappa)^{q-1}(1-a)^{1-q}=1+(q-1) \rho \\
\mathrm{d} \lambda: & \int x f_{t}(x) F_{t}(\mathrm{~d} x)=0 \\
\mathrm{~d} \rho: & \int f_{t}(x) v_{t}(\mathrm{~d} x)-a-\kappa=0
\end{array}
$$


From the first Equation in (64) we are going to have

$$
\begin{aligned}
& f^{q-1}=(1-(q-1) \rho)\left(1+\frac{(q-1) \lambda x}{1+(q-1) \rho}\right) \\
& \text { Let } \Gamma=\frac{(q-1) \lambda}{1+(q-1) \rho} \\
& f=[(1-(q-1) \rho)(1+\Gamma x)]^{\frac{1}{q-1}}
\end{aligned}
$$

From second Equation in (64) make $\kappa$ the subject

$$
\kappa=1-a-\left(\frac{1+(q-1) \rho}{(1-a)^{1-q}}\right)^{\frac{1}{q-1}}
$$

Substitute (65) and (66) into the fourth Equation in (64) and make $\rho$ the subject.

$$
\rho=\frac{1}{q-1}\left(\frac{1}{1-a+\int(1+\Gamma x)^{\frac{1}{q-1}} \nu_{t}(\mathrm{~d} x)}\right)^{q-1}-\frac{1}{q-1}
$$

Substitute the Equation (67) into Equation (66)

$$
f_{t}(x)=\frac{(1+\Gamma x)^{\frac{1}{q-1}}}{1-a+\int(1+\Gamma x)^{\frac{1}{q-1}} \nu_{t}(\mathrm{~d} x)}
$$

From Equation (65), $\Gamma=\frac{(q-1) \lambda}{1+(q-1) \rho}$. That means $\Gamma \propto \lambda$, Therefore $\Gamma=k \lambda$ and $k=\frac{(q-1)}{1+(q-1) \rho}$. If we assume $k=1=\frac{(q-1) \lambda}{1+(q-1) \rho}$. Then $\Gamma=\lambda$

Then we are going to have

$$
f_{t}(x)=\frac{(1+\lambda x)^{\frac{1}{q-1}}}{1-a+\int(1+\lambda x)^{\frac{1}{q-1}} v_{t}(\mathrm{~d} x)}
$$

So if you multiply the above Equation (69) by $v_{t}(\mathrm{~d} x)$ and integrate both sides with respect to it we get:

$$
\int f_{t}(x) v_{t}(\mathrm{~d} x)=\frac{\int(1+\lambda x)^{\frac{1}{q-1}} v_{t}(\mathrm{~d} x)}{1-a+\int(1+\lambda x)^{\frac{1}{q-1}} v_{t}(\mathrm{~d} x)}
$$

If you add Equation (69) and and Equation (70) we get $W=f_{t}(x)+\int f_{t}(x) v_{t}(\mathrm{~d} x)$ which is equal to $W=f_{t}(x)+\tilde{f}_{t}(x)$ and this equation is not equal to given $W=f-1+\frac{\hat{f}-a}{1-a}$.

This function $W=f_{t}(x)+\int f_{t}(x) v_{t}(\mathrm{~d} x)$ gives us

$$
\tilde{W}=\frac{(1+\lambda x)^{\frac{1}{q-1}}+\int(1+\lambda x)^{\frac{1}{q-1}} v_{t}(\mathrm{~d} x)}{1-a+\int(1+\lambda x)^{\frac{1}{q-1}} v_{t}(\mathrm{~d} x)} \text {. }
$$


Since $W=f-1+\frac{\hat{f}-a}{1-a}$, in order to get the required minimal entropy-Hellinger sigma martingale density we need to introduce the new function

$$
m=f_{t}-1 \in \tilde{\mathcal{P}} \text { where } f=\frac{(1+\lambda x)^{\frac{1}{q-1}}}{1-a \int(1+\lambda x)^{\frac{1}{q-1}} v_{t}(\mathrm{~d} x)} .
$$

Therefore

$$
m=f_{t}-1=\frac{(1+\lambda x)^{\frac{1}{q-1}}}{1-a+\int(1+\lambda x)^{\frac{1}{q-1}} v_{t}(\mathrm{~d} x)}-1
$$

Multiply both sides by $v_{t}(\mathrm{~d} x)$ and integrate both sides. Then we are going to get:

$$
\frac{\int f_{t}(x) v_{t}(\mathrm{~d} x)-a}{1-a}=\frac{\int(1+\lambda x)^{\frac{1}{q-1}} v_{t}(\mathrm{~d} x)-a}{1-a+\int(1+\lambda x)^{\frac{1}{q-1}} v_{t}(\mathrm{~d} x)}
$$

By adding Equation (71) and Equation (72) we get the required minimal entropy-Hellinger sigma martingale density Equation (54).

As we have seen on entropy-Hellinger process of order one, a measurable function $W$ in Jacod decomposition depends $U \in \tilde{P}$. We proved that, when we have another version of $U$ it will change a measurable function $W$, an expression of entropy-Hellinger process as well as $\tilde{W}$. In order to get a measurable function $\tilde{W}$ for an equation of minimal entropy-Hellinger sigma martingale density even in this entropy process of order $q$ we introduce the new function $m=f_{t}-1 \in \tilde{\mathcal{P}}$ and get a required $W^{(q)}(t, x)=\frac{\left(1+\tilde{\lambda}_{t}^{\mathrm{T}} x\right)^{\frac{1}{q-1}}-1}{1-a_{t}+\int\left(1+\tilde{\lambda}_{t}^{\mathrm{T}} x\right)^{\frac{1}{q-1}} v_{t}(\mathrm{~d} x)}$. Also in our case we find out $\beta=\lambda$ which is different compare to [4] where $\beta=\frac{1}{q-1} \lambda$.

\section{An Expression of Entropy Hellinger Process for a Positive Sigma Martingale of Order Zero}

If $N \in \mathcal{M}_{0, \text { loc }}(P)$ be such that $(1+\Delta N)>0$ and $q \neq 1$ such that the nondecreasing adapted process

$$
V_{t}^{0}(N)=\frac{1}{2}\left\langle N^{c}\right\rangle_{t}+\sum_{0<s \leq t} \phi_{0}\left(\Delta N_{s}\right), \quad 0 \leq t \leq T
$$

is locally integrable, then the Hellinger process $H^{(0)}(N, P)$ of order $q=0$ is the dual predictable projection of $V_{t}^{0}(N)$ with respect to $P$.

The function $\phi_{0}(x)$ on the above Equation (73) is given as

$$
\phi_{0}(x)= \begin{cases}x-\log (1+x), & \text { if } x>-1 \text { and } q=0 \\ +\infty & \text { otherwise }\end{cases}
$$

The set of sigma martingale densities which we are interested in: 


$$
\begin{aligned}
\mathcal{Z}_{\text {loc }}^{e}(S)= & \left\{Z=\mathcal{E}(N) \geq 0 \mid N \in \mathcal{M}_{\text {loc }}(P): Z>0 Z \log Z\right. \text { locally integrable, } \\
& \left.Z S \in \mathcal{M}_{\sigma}(P), \phi_{0}(\Delta N) \in L_{\text {loc }}^{1}\right\}
\end{aligned}
$$

As we have seen in the entropy-Hellinger process of order one: The entropy-Hellinger process of $N$ with respect to probability measure $P\left(h^{(0)}(N, P)\right)$ is the same as of sigma density $Z \in \mathcal{Z}_{\text {loc }}^{e}(q=0)$ denoted by $\left(h_{t}^{(0)}(Z, P)\right)$ and also is the same as of equivalent probability measure $\left(Q \in \mathbb{P}_{a}\right)$ denoted by $\left(h_{t}^{(0)}(Q, P)\right)$.

Proposition 5 (The entropy-Hellinger process of order zero)

The Hellinger process of order 0 for $N$ when $W=f-1+\frac{\hat{f}-1}{1-a} \mathbf{1}_{\{a<1\}}$ is equal to

$$
\begin{aligned}
h^{(0)}(N, P)= & \frac{1}{2} \beta^{\mathrm{T}} c \beta \cdot A+\int_{0}^{T} \int_{\mathbb{R}}[(f-1)-\ln f] F_{t}(\mathrm{~d} x)(\mathrm{d} A) \\
& +\sum_{0<s \leq t}\left(-(\hat{f}-a)-(1-a) \ln \left(1-\frac{\hat{f}-a}{1-a}\right)\right) \\
& +f \mathcal{M}_{\mu}^{P}\left(\frac{g}{f}-\ln \left(1+\frac{g}{f}\right) \mid \tilde{P}\right) \star v+L .
\end{aligned}
$$

Proof

From Jacod decomposition $\Delta N_{t}^{\prime}=\Delta N_{t}^{\prime} \mathbf{1}_{\{\Delta S=0\}}$,

We have the following assertions

$$
g \mathbf{1}_{f=0}=0 \text { and } \Delta N^{\prime} \mathbf{1}_{\frac{\hat{f}-a}{1-a}=0}
$$

From Equation (9)

$$
\Delta N=(f+g-1) \mathbf{1}_{\Delta S \neq 0}+\left(\Delta N^{\prime}-\frac{\hat{f}-a}{1-a}\right) \mathbf{1}_{\Delta S=0}
$$

Also from (12) we have

$$
\begin{gathered}
1+\Delta N=(f+g) \mathbf{1}_{\Delta S \neq 0}+\left(1+\Delta N^{\prime}-\frac{\hat{f}-a}{1-a}\right) \mathbf{1}_{\Delta S=0} \\
(f+g) \geq 0, \quad 1+\Delta N^{\prime}-\frac{\hat{f}-a}{1-a} \geq 0
\end{gathered}
$$

By taking the conditional expectation in the first equation in Equation (75) with respect to $\tilde{P}$ and predictable projection in the second Equation in (75) we are going to have

$$
f \geq 0, \quad 1-\frac{\hat{f}-a}{1-a} \geq 0
$$

From Equation (73) on the RHS we have

$$
\begin{aligned}
\phi_{0}\left(\Delta N_{s}\right)= & \sum_{0<s \leq t}(\Delta N-\ln (1+\Delta N)) \\
= & ((f+g-1)-\ln (f+g)) \star \mu \\
& +\left(\left(\Delta N^{\prime}-\frac{\hat{f}-a}{1-a}\right)-\ln \left(1+\Delta N^{\prime}-\frac{\hat{f}-a}{1-a}\right)\right) \mathbf{1}_{\Delta S=0}
\end{aligned}
$$


By using the assertion above

$$
\begin{aligned}
V(N, P)= & \frac{1}{2} \beta^{\mathrm{T}} c \beta \cdot A+((f-1)-\ln f) \star \mu \\
& +\sum_{0<s \leq t}\left(\left(-\frac{\hat{f}-a}{1-a}\right)-\ln \left(1-\frac{\hat{f}-a}{1-a}\right)\right) \mathbf{1}_{\Delta S=0} \\
& +f\left(\frac{g}{f}-\ln \left(1+\frac{g}{f}\right)\right) \star \mu+\frac{1}{2}\left\langle N^{c^{\prime}}\right\rangle \\
& +\sum_{0<s \leq t}\left(1-\frac{\hat{f}-a}{1-a}\right)\left[\left(\frac{\Delta N^{\prime}}{\left.1-\frac{\hat{f}-a}{1-a}\right)}\right)-\left(1+\frac{\Delta N^{\prime}}{1-\frac{\hat{f}-a}{1-a}}\right)\right] \mathbf{1}_{\Delta S=0} \\
& +g \ln f \star \mu+\sum_{0<s \leq t} \ln \left(1-\frac{\hat{f}-a}{1-a}\right) \Delta N^{\prime}
\end{aligned}
$$

The dual predictable projection of the last two terms in the RHS of the Equation (77) will vanish due to the duality predictable projection properties of local martingal $N$ and $g$.

Let $L$ be dual predictable projection of the following equation

$$
K=\frac{1}{2}\left\langle N^{c^{\prime}}\right\rangle+\sum_{0<s \leq t}\left(1-\frac{\hat{f}-a}{1-a}\right)\left[\left(\frac{\Delta N^{\prime}}{1-\frac{\hat{f}-a}{1-a}}\right)-\left(1+\frac{\Delta N^{\prime}}{1-\frac{\hat{f}-a}{1-a}}\right)\right] \mathbf{1}_{\Delta S=0}
$$

Taking the dual predictable projection of the remaining terms we are going to have.

$$
\begin{aligned}
h^{0}(N, P)= & \frac{1}{2} \beta^{\mathrm{T}} c \beta \cdot A+((f-1)-\ln f) \star v \\
& +\sum_{0<s \leq t}\left(\left(-\frac{\hat{f}-a}{1-a}\right)-\ln \left(1-\frac{\hat{f}-a}{1-a}\right)\right)(1-a) \\
& +f \mathcal{M}_{\mu}^{P}\left(\frac{g}{f}-\ln \left(1+\frac{g}{f}\right) \mid \tilde{P}\right) \star v+L
\end{aligned}
$$

Which is the same as (74)

The minimization problem of $\min _{Z \in \mathcal{Z}_{e, l o c}} h^{E}(Z, P)$ is equivalent to minimize the Hellinger process over the set of densities that have the following predictable representation $\left(g=0\right.$ and $\left.N^{\prime}=0\right)$.

Therefore we are going to have the following minimization entropy-Hellinger process.

$$
\begin{aligned}
& h^{0}(N, P) \\
& =\frac{1}{2} \beta^{\mathrm{T}} c \beta \cdot A+((f-1)-\ln f) \star v \\
& +\sum_{0<s \leq t}\left(-(\hat{f}-a)-(1-a) \ln \left(1-\frac{\hat{f}-a}{1-a}\right)\right)
\end{aligned}
$$




\section{Solution of Minimization Problem Based on Entropy Hellinger Process of Sigma Martingale Density of Order Zero}

By distinguishing the cases where $\Delta A=0$ and the case where $\Delta A \neq 0$, this problem can be split into the following two minimization problems. The first problem $(\Delta A=0)$ is defined by

$$
\int \frac{1}{2} \beta^{\mathrm{T}} c \beta \mathrm{d} A+\int((f-1)-\ln f) F_{t} \mathrm{~d} A
$$

where the minimization is over all couples $(\beta, f)$ satisfying

$$
b+c \beta+\int(x(f+1)-h(x)) F_{t}(\mathrm{~d} x)=0
$$

The second problem $(\Delta A \neq 0)$ is defined as follows

$$
((f-1)-\ln f) \star v+\sum_{0<s \leq t}\left(-(\hat{f}-a)-(1-a) \ln \left(1-\frac{\hat{f}-a}{1-a}\right)\right)
$$

where the minimization is over the functional $f$ such that

$$
\int x f v_{t}(\mathrm{~d} x)=0
$$

The conditions (81) and (83) correspond to the conditions given in above theorem and conditions given for a local martingale to be a sigma martingale [5].

\subsection{Solving Euler-Lagrange Equation of the First Problem}

Euler-Lagrange equation of the first problem due to Equation (80) and Equation (81)

$$
\begin{aligned}
& L(\beta, f)= \int \frac{1}{2} \beta^{\mathrm{T}} c \beta \mathrm{d} A+\int((f-1)-\ln f) F_{t}(\mathrm{~d} x) \mathrm{d} A \\
&-\lambda\left(\int b \mathrm{~d} A+\int c \beta \mathrm{d} A+\int(x(f+1)-h(x)) F_{t}(\mathrm{~d} x) \mathrm{d} A\right) \\
&= 0 \\
& L(\beta, f)= \frac{1}{2} \beta^{\mathrm{T}} c \beta+\int((f-1)-\ln f) F_{t}(\mathrm{~d} x) \\
&-\lambda\left(b+c \beta+\int(x(f+1)-h(x)) F_{t}(\mathrm{~d} x)\right) \\
&=0 \quad \mathrm{~d} \beta: \beta=\lambda \\
& \mathrm{d} f: f=\frac{1}{1-\lambda x}
\end{aligned}
$$

Therefore the description of $\tilde{\beta}$ and $\tilde{f}$ due to Equation (84) is equal to

$$
\tilde{\beta}=\tilde{\lambda} \mathbf{1}_{\{\triangle A=0\}}, \quad \tilde{f} \mathbf{1}_{\{\triangle A=0\}}=\left(\frac{1}{1-\lambda X}\right) \mathbf{1}_{\{\triangle A=0\}}
$$

\subsection{Solving Euler-Lagrange Equation of the Second Problem}

The Lagrangian for this minimization problem due to Equations (82) and (83) is 
given by

$$
\begin{aligned}
& L(f, \phi, \lambda, \alpha)=\int((f-1)-\ln f) v_{t}(\mathrm{~d} x)+\sum_{0<s \leq t}\left[-\phi-(1-a) \ln \left(1-\frac{\phi}{1-a}\right)\right] \\
& -\lambda\left(\int x f_{t}(x) v_{t}(\mathrm{~d} x)\right)-\alpha\left(\int f_{t}(x) v_{t}(\mathrm{~d} x)-a-\phi\right) \\
& =0 \\
& \mathrm{~d} f: f_{t}(x)=\frac{1}{1-\lambda x-\alpha} \\
& \mathrm{d} \phi: \frac{1-a}{(1-a)-\phi}=1-\alpha \\
& \mathrm{d} \lambda: \int x f_{x} v_{d x}=0 \\
& \mathrm{~d} \alpha: \int f_{t}(x) v_{t}(\mathrm{~d} x)-a-\phi=0
\end{aligned}
$$

Substitute the first Equation of (86) into the third Equation of (86)

$$
\int \frac{x}{1-\lambda x-\alpha} v_{t}(\mathrm{~d} x)=0
$$

From the second Equation (86) make $\phi$ the subject

$$
\phi=\frac{(1-a)(-\alpha)}{1-\alpha}
$$

Substitute Equation (88) into the fourth Equation (86) and make $\alpha$ the subject

$$
\begin{aligned}
& \int f_{t}(x) v_{t}(\mathrm{~d} x)-a-\left(\frac{(1-a)(-\alpha)}{1-\alpha}\right)=0 \\
& \alpha=\frac{\int f_{t}(x) v_{t}(\mathrm{~d} x)-a}{\int f_{t}(x) v_{t}(\mathrm{~d} x)-1}
\end{aligned}
$$

Substitute Equation (89) into the first Equation (86)

$$
f_{t}(x)=\frac{\int f_{t}(x) v_{t}(\mathrm{~d} x)-1}{(1-\lambda x)\left(\int f_{t}(x) v_{t}(\mathrm{~d} x)-1\right)-\left(\int f_{t}(x) v_{t}(\mathrm{~d} x)-a\right)}
$$

Multiply both sides of Equation (90) by $v_{t}(\mathrm{~d} x)$ and integrate the equation

$$
\begin{aligned}
& \int f_{t}(x) v_{t}(\mathrm{~d} x)=\frac{a}{1-\lambda x-\left[\frac{\int f_{t}(x) v_{t}(\mathrm{~d} x)-a}{\int f_{t}(x) v_{t}(\mathrm{~d} x)-1}\right]} \\
& \frac{\int f_{t}(x) v_{t}(\mathrm{~d} x)}{a}=\frac{\int f_{t}(x) v_{t}(\mathrm{~d} x)-1}{(1-\lambda x)\left(\int f_{t}(x) v_{t}(\mathrm{~d} x)-1\right)-\left(\int f_{t}(x) v_{t}(\mathrm{~d} x)-a\right)}
\end{aligned}
$$

If we add Equations (90) and Equation (91) on LHS we are going to have $W=f_{t}(x)+\frac{\int f_{t}(x) v_{t}(\mathrm{~d} x)}{a}$ which is equal to $W=f_{t}(x)+\frac{\hat{f}_{t}(x)}{a}$ and this equation gives us 


$$
\begin{aligned}
\hat{W}= & \frac{\int f_{t}(x) v_{t}(\mathrm{~d} x)-1}{(1-\lambda x)\left(\int f_{t}(x) v_{t}(\mathrm{~d} x)-1\right)-\left(\int f_{t}(x) v_{t}(\mathrm{~d} x)-a\right)} \\
& +\frac{\int f_{t}(x) v_{t}(\mathrm{~d} x)-1}{(1-\lambda x)\left(\int f_{t}(x) v_{t}(\mathrm{~d} x)-1\right)-\left(\int f_{t}(x) v_{t}(\mathrm{~d} x)-a\right)}
\end{aligned}
$$

This is equal to

$$
\hat{W}=2 \frac{\int f_{t}(x) v_{t}(\mathrm{~d} x)-1}{(1-\lambda x)\left(\int f_{t}(x) v_{t}(\mathrm{~d} x)-1\right)-\left(\int f_{t}(x) v_{t}(\mathrm{~d} x)-a\right)}
$$

But we know $W=f-1+\frac{\hat{f}-a}{1-a}$

Therefore let

$$
\begin{gathered}
m=f_{t}(x)-1 \in \tilde{\mathcal{P}} \text { where } f_{t}(x)=\frac{1}{1-\lambda x-\left[\frac{\int f_{t}(x) v_{t}(\mathrm{~d} x)-a}{\int f_{t}(x) v_{t}(\mathrm{~d} x)-1}\right]} \\
m=f_{t}(x)-1=\frac{1}{1-\lambda x-\left[\frac{\int f_{t}(x) v_{t}(\mathrm{~d} x)-a}{\int f_{t}(x) v_{t}(\mathrm{~d} x)-1}\right]}-1
\end{gathered}
$$

From Equation (92)

$$
f_{t}(x)-1=\frac{\left(\int f_{t}(x) v_{t}(\mathrm{~d} x)-1\right)-\left((1-\lambda x)\left(\int f_{t}(x) v_{t}(\mathrm{~d} x)-1\right)-\left(\int f_{t}(x) v_{t}(\mathrm{~d} x)-a\right)\right)}{(1-\lambda x)\left(\int f_{t}(x) v_{t}(\mathrm{~d} x)-1\right)-\left(\int f_{t}(x) v_{t}(\mathrm{~d} x)-a\right)}
$$

Multiply both sides of Equation (92) by $v_{t}(\mathrm{~d} x)$ and integrate both sides

$$
\int f_{t}(x) v_{t}(\mathrm{~d} x)-a=\frac{a\left[\int f_{t}(x) v_{t}(\mathrm{~d} x)-1\right]}{(1-\lambda x)\left(\int f_{t}(x) v_{t}(\mathrm{~d} x)-1\right)-\left(\int f_{t}(x) v_{t}(\mathrm{~d} x)-a\right)}-a
$$

If we put the common denominator in the above equation we are going to have

$$
\begin{gathered}
\int f_{t}(x) v_{t}(\mathrm{~d} x)-a=\frac{a\left(\lambda x-\frac{1}{a}\left(1+\frac{1-a}{\int f_{t}(x) v_{t}(\mathrm{~d} x)-1}\right)\right)}{\left(-\lambda x-\frac{1-a}{\int f_{t}(x) v_{t}(\mathrm{~d} x)-1}\right)} \\
\frac{\int f_{t}(x) v_{t}(\mathrm{~d} x)-a}{1-a}=\frac{\frac{a}{1-a}\left(\lambda x-\frac{1}{a}\left(1+\frac{1-a}{\int f_{t}(x) v_{t}(\mathrm{~d} x)-1}\right)\right)}{\left(-\lambda x-\frac{1-a}{\int f_{t}(x) v_{t}(\mathrm{~d} x)-1}\right)}
\end{gathered}
$$

If you add LHS Equations (93) and (95) we are going to have

$$
W=f_{t}(x)-1+\frac{\int f_{t}(x) v_{t}(\mathrm{~d} x)-a}{1-a}
$$

And if you add the RHS of Equations (93) and (95) we are going to have 


$$
\begin{aligned}
\hat{W}= & \frac{\left(\int f_{t}(x) v_{t}(\mathrm{~d} x)-1\right)-\left((1-\lambda x)\left(\int f_{t}(x) v_{t}(\mathrm{~d} x)-1\right)-\left(\int f_{t}(x) v_{t}(\mathrm{~d} x)-a\right)\right)}{(1-\lambda x)\left(\int f_{t}(x) v_{t}(\mathrm{~d} x)-1\right)-\left(\int f_{t}(x) v_{t}(\mathrm{~d} x)-a\right)} \\
& +\frac{\frac{a}{1-a}\left(\lambda x-\frac{1}{a}\left(1+\frac{1-a}{\int f_{t}(x) v_{t}(\mathrm{~d} x)-1}\right)\right)}{\left(-\lambda x-\frac{1-a}{\int f_{t}(x) v_{t}(\mathrm{~d} x)-1}\right)}
\end{aligned}
$$

The result is difference compared with the order one and order is not equal to one. This is because even after we introduce a function $m=f_{t}-1 \in \tilde{\mathcal{P}}$ in order to get $\hat{W}$ for minimal entropy Hellinger sigma martingale density, we fail to get a required results.

Therefore: For order zero it is possible to get the equation of $W$ but it is not possible to get a required $\hat{W}$ for the minimal entropy-Hellinger sigma martingale density.

\section{Conclusions and Recommendations}

In the process of forming expressions of Entropy-Hellinger processes of order one, order $q$ and order zero the researchers used a local martingale jump equation of Jacod decomposition. As we know Jacod decomposition has known parameters $\left(\beta, W, g, N^{\prime}\right)$. A measurable function $W=U+\frac{\hat{U}}{1-a}$ where $U \in \tilde{\mathcal{P}}$. If we have a new version of $U$ with the conditions that $\Delta N>-1$ then also

$U>-1$. If we set $U=f-1$, then we are going to have $W=f-1+\frac{\hat{f}-a}{1-a} \mathbf{1}_{\{a<1\}}$. In this research work, we provide and prove other expressions of entropy-Hellinger processes for a positive sigma martingale of order one, order $q$ and order zero. Furthermore, we show how measurable functions $W$ and $\tilde{W}$ change during our minimizations solutions and we introduce a function $m=f_{t}-1 \in \tilde{\mathcal{P}}$ in order to get a required equation of minimal entropy-Hellinger sigma martingale density. However, the results is different to the minimization of entropy-Hellinger process of order zero because it is possible to get an equation of measurable function $W$ after an introduction of function $m=f_{t}-1 \in \tilde{\mathcal{P}}$ but it is not possible to get an equation of measurable function $\tilde{W}$.

This study is based on a proposed dynamic method of finding an equivalent sigma martingale measure and/or density by using entropy-Hellinger process. Since we show it is possible to have other expressions of entropy-Hellinger processes of all orders. For future studies we recommend other forms of expressions of entropy-Hellinger processes to be considered. This will be possible if new versions of parameters of Jacod decomposition are set under needed conditions.

\section{Acknowledgements}

We acknowledge the African Union through the Pan African University, Insti- 
tute of Basic Sciences, Technology and Innovation for its consideration support.

\section{Conflicts of Interest}

The authors declare no conflicts of interest regarding the publication of this paper.

\section{References}

[1] Choulli, T. and Hurd, T.R. (2001) The Role of Hellinger Processes in Mathematical Finance. Entropy, 3, 150-161. https://doi.org/10.3390/e3030150

[2] Choulli, T. and Stricker, C. (2009) Comparing the Minimal Hellinger Martingale Measure of Order q to the q-Optimal Martingale Measure. Stochastic Processes and Their Applications, 119, 1368-1385. https://doi.org/10.1016/j.spa.2008.07.002

[3] Choulli, T. and Stricker, C. (2006) More on Minimal Entropy-Hellinger Martingale Measure. Mathematical Finance: An International Journal of Mathematics, Statistics and Financial Economics, 16, 1-19. https://doi.org/10.1111/j.1467-9965.2006.00258.x

[4] Choulli, T., Stricker, C. and Li, J. (2007) Minimal Hellinger Martingale Measures of Order q. Finance and Stochastics, 11, 399-427. https://doi.org/10.1007/s00780-007-0039-3

[5] Ma, J.F. (2013) Minimal Hellinger Deflators and HARA forward Utilities with Applications: Hedging with Variable Horizon.

[6] Kabanov, Y., Rutkowski, M. and Zariphopoulou, T. (2013) Inspired by Finance: The Musiela Festschrift. Springer Science \& Business Media, Berlin. https://doi.org/10.1007/978-3-319-02069-3

[7] Choulli, T. and Schweizer, M. (2011) Stability of Sigma-Martingale Densities in L Log L under an Equivalent Change of Measure. Swiss Finance Institute Research Paper, (11-67). https://doi.org/10.2139/ssrn.1986855

[8] Choulli, T. and Schweizer, M. (2015) Locally Phi-Integrable Sigma-Martingale Densities for General Semimartingales. Swiss Finance Institute Research Paper, (15-15). https://doi.org/10.2139/ssrn.2606674

[9] Harrison, J.M. and Kreps, D.M. (1979) Martingales and Arbitrage in Multiperiod Securities Markets. Journal of Economic Theory, 20, 381-408. https://doi.org/10.1016/0022-0531(79)90043-7

[10] Harrison, J.M. and Pliska, S.R. (1981) Martingales and Stochastic Integrals in the Theory of Continuous Trading. Stochastic Processes and Their Applications, 11, 215-260. https://doi.org/10.1016/0304-4149(81)90026-0

[11] Kallsen J. (2002) Utility-Based Derivative Pricing in Incomplete Markets. In: Mathematical Finance Bachelier Congress, Springer, Berlin, 313-338. https://doi.org/10.1007/978-3-662-12429-1 15

[12] Follmer, H. and Schweizer, M. (2010) Minimal Martingale Measure. Encyclopedia of Quantitative Finance. https://doi.org/10.1002/9780470061602.eqf04015

[13] Schweizer, M. (1995) On the Minimal Martingale Measure and the Mollmer-Schweizer Decomposition. Stochastic Analysis and Applications, 13, 573-599. https://doi.org/10.1080/07362999508809418

[14] Schweizer, M. (1999) A Minimality Property of the Minimal Martingale Measure. Statistics \& Probability Letters, 42, 27-31.

https://doi.org/10.1016/S0167-7152(98)00180-1 
[15] Follmer, H. and Schweizer, M. (1991) Hedging of Contingent Claims under Incomplete Information. Applied Stochastic Analysis, 5, 19-31.

[16] Delbaen, F., Schachermayer, W., et al. (1996) The Variance-Optimal Martingale Measure for Continuous Processes. Bernoulli, 2, 81-105. https://doi.org/10.2307/3318570

[17] Schweizer, M., et al. (1996) Approximation Pricing and the Variance-Optimal Martingale Measure. The Annals of Probability, 24, 206-236. https://doi.org/10.1214/aop/1042644714

[18] Frittelli, M. (2000) The Minimal Entropy Martingale Measure and the Valuation Problem in Incomplete Markets. Mathematical Finance, 10, 39-52. https://doi.org/10.1111/1467-9965.00079

[19] Miyahara, Y. (1996) Canonical Martingale Measures of Incomplete Assets Markets. Probability Theory and Mathematical Statistics, Tokyo, 26-30 July 1995, 343-352.

[20] Miyahara, Y. (1999) Minimal Entropy Martingale Measures of Jump Type Price Processes in Incomplete Assets Markets. Asia-Pacific Financial Markets, 6, 97-113. https://doi.org/10.1023/A:1010062625672

[21] Miyahara, Y. (2001) [Geometric Levy Process \& MEMM] Pricing Model and Related Estimation Problems. Asia-Pacific Financial Markets, 8, 45-60. https://doi.org/10.1023/A:1011445109763

[22] Goll, T. and Ruschendorf, L. (2001) Minimax and Minimal Distance Martingale Measures and Their Relationship to Portfolio Optimization. Finance and Stochastics, 5, 557-581. https://doi.org/10.1007/s007800100052

[23] Bellini, F. and Frittelli, M. (2002) On the Existence of Minimax Martingale Measures. Mathematical Finance, 12, 1-21. https://doi.org/10.1111/1467-9965.00001

[24] Choulli, T. and Stricker, C. (2005) Minimal Entropy-Hellinger Martingale Measure in Incomplete Markets. Mathematical Finance: An International Journal of Mathematics, Statistics and Financial Economics, 15, 465-490. https://doi.org/10.1111/j.1467-9965.2005.00229.x

[25] Grandits, P. (2000) On Martingale Measures for Stochastic Processes with Independent Increments. Theory of Probability \& Its Applications, 44, 39-50. https://doi.org/10.1137/S0040585X97977355

[26] Davis, M.H.A. (1997) Option Pricing in Incomplete Markets. Mathematics of Derivative Securities, 15, 216-226.

[27] Karatzas, I., Lehoczky, J.P. and Shreve, S.E. (1987) Optimal Portfolio and Consumption Decisions for a Small Investor on a Finite Horizon. SIAM Journal on Control and Optimization, 25, 1557-1586. https://doi.org/10.1137/0325086

[28] Kramkov, D. and Schachermayer, W. (1999) The Asymptotic Elasticity of Utility Functions and Optimal Investment in Incomplete Markets. Annals of Applied Probability, 9, 904-950. https://doi.org/10.1214/aoap/1029962818

[29] Kallsen, J. (1999) A Utility Maximization Approach to Hedging in Incomplete Markets. Mathematical Methods of Operations Research, 50, 321-338. https://doi.org/10.1007/s001860050100

[30] Becherer, D. (2001) Rational Hedging and Valuation with Utility-Based Preferences.

[31] Jacod, J. (2006) Calcul stochastique et problemes de martingales, Volume 714. Springer, Berlin. 